\title{
Hydration-Assisted Sorption of a Probe Organic Compound at Different Peat Hydration Levels: The Link Solvation Model
}

\section{SUPPORTING INFORMATION}

\author{
E.R. Graber*, L. Tsechansky, and M. Borisover \\ Institute of Soil, Water and Environmental Sciences \\ The Volcani Center \\ Agricultural Research Organization \\ Bet Dagan, 50250, Israel \\ ergraber@agri.gov.il
}

*Corresponding Author: Tel. 972-3-968-3307; Fax. 972-3-960-4017 
Table S1. Experimental conditions for sorption experiments

\begin{tabular}{|l|l|l|l|l|l|}
\hline $\begin{array}{l}\text { Moisture } \\
\text { Content } \\
(\%)\end{array}$ & $\begin{array}{l}\mathrm{H}_{2} \mathrm{O} \\
\text { activity }\end{array}$ & $\begin{array}{l}\text { Solid:liquid } \\
\text { ratio } \\
(\mathrm{g} / \mathrm{mL})\end{array}$ & $\begin{array}{l}\text { Time (day) } \\
\text { isotherm/kinetics } \\
\text { /equilibrium }^{\mathrm{a}}\end{array}$ & $\begin{array}{l}\text { Sorbed } \\
\text { portion of } \\
\text { total }(\%)\end{array}$ & $\begin{array}{l}\text { Mean loss } \\
(\mathrm{st} \text { dev. }) \\
(\%)\end{array}$ \\
\hline 3.2 & 0.07 & $1: 20$ & $28 / 42 / 14$ & $10-55$ & $2(4)$ \\
\hline 7.9 & 0.24 & $1: 20$ & $28 / 42 / 14$ & $15-65$ & $0(7)$ \\
\hline 12.1 & 0.41 & $1: 30$ & $28 / 42 / 21$ & $20-70$ & $0(6)$ \\
\hline 16.0 & 0.55 & $1: 30$ & $28 / 42 / 21$ & $45-85$ & $0(6)$ \\
\hline 20.1 & 0.68 & $1: 40$ & $28 / 42 / 21$ & $35-80$ & $0(7)$ \\
\hline 25.1 & 0.80 & $1: 40$ & $28 / 42 /$ nd & $35-80$ & $1(4)$ \\
\hline 30.0 & 0.89 & $1: 40$ & $28 / 42 / 21$ & $35-75$ & $-1(7)$ \\
\hline 34.9 & 0.96 & $1: 40$ & $28 / 42 / \mathrm{nd}$ & $35-85$ & $0(6)$ \\
\hline 40.3 & 0.99 & $1: 40$ & $28 / 42 / \mathrm{nd}$ & $40-90$ & $0(6)$ \\
\hline
\end{tabular}

atime in days elapsed for measuring equilibrium isotherm/ maximum time reached in kinetics series/ time required to reach equilibrium ${ }^{\mathrm{b}}$ nd means not determined

Table S2. Isotherm Freundlich parameters.

\begin{tabular}{|l|l|l|l|l|}
\hline $\mathbf{H}_{\mathbf{2}} \mathbf{O}$ Activity & $\begin{array}{l}\text { Moisture Content } \\
\text { (w/w \%) }\end{array}$ & $\begin{array}{l}\mathbf{K}_{\mathbf{f}} \\
\text { (standard error) }\end{array}$ & $\begin{array}{l}\boldsymbol{n} \\
\text { (standard error) }\end{array}$ & $\mathbf{R}^{2}$ \\
\hline 0.07 & 3.2 & $52(8)$ & $0.544(0.022)$ & 0.995 \\
\hline 0.24 & 7.9 & $58(8)$ & $0.605(0.019)$ & 0.997 \\
\hline 0.41 & 12.1 & $94(20)$ & $0.640(0.032)$ & 0.993 \\
\hline 0.55 & 16.0 & $214(12)$ & $0.701(0.008)$ & 0.999 \\
\hline 0.68 & 20.1 & $347(65)$ & $0.681(0.028)$ & 0.996 \\
\hline 0.80 & 25.1 & $327(54)$ & $0.694(0.025)$ & 0.997 \\
\hline 0.89 & 30.0 & $279(30)$ & $0.712(0.016)$ & 0.999 \\
\hline 0.96 & 34.9 & $204(18)$ & $0.751(0.013)$ & 0.999 \\
\hline 0.99 & 40.3 & $191(36)$ & $0.764(0.028)$ & 0.998 \\
\hline
\end{tabular}




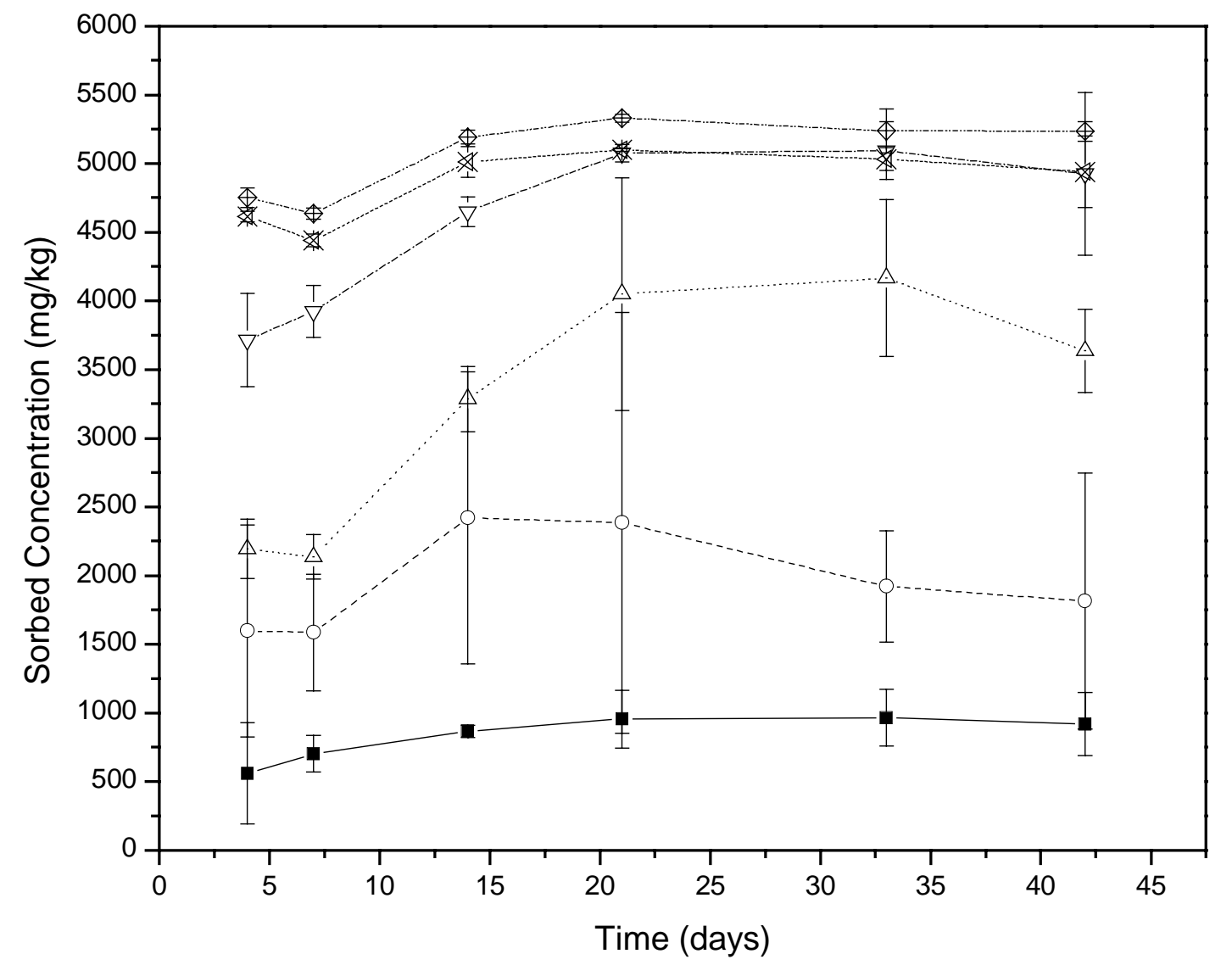

Moisture Content (\%)



Fig S1. Kinetics of phenol sorption on Pahokee peat at 6 different hydration levels, indicated as moisture content in the figure. Equivalent water activities can be found in Tables S1 and S2. 




Fig. S2. Relationship between Freundlich exponent $n$ and water activity for the nine different systems. Dotted lines show 95\% confidence interval of the best fit line. Freundlich model parameters and fitting statistics are found in Table S2, Supporting Information. 

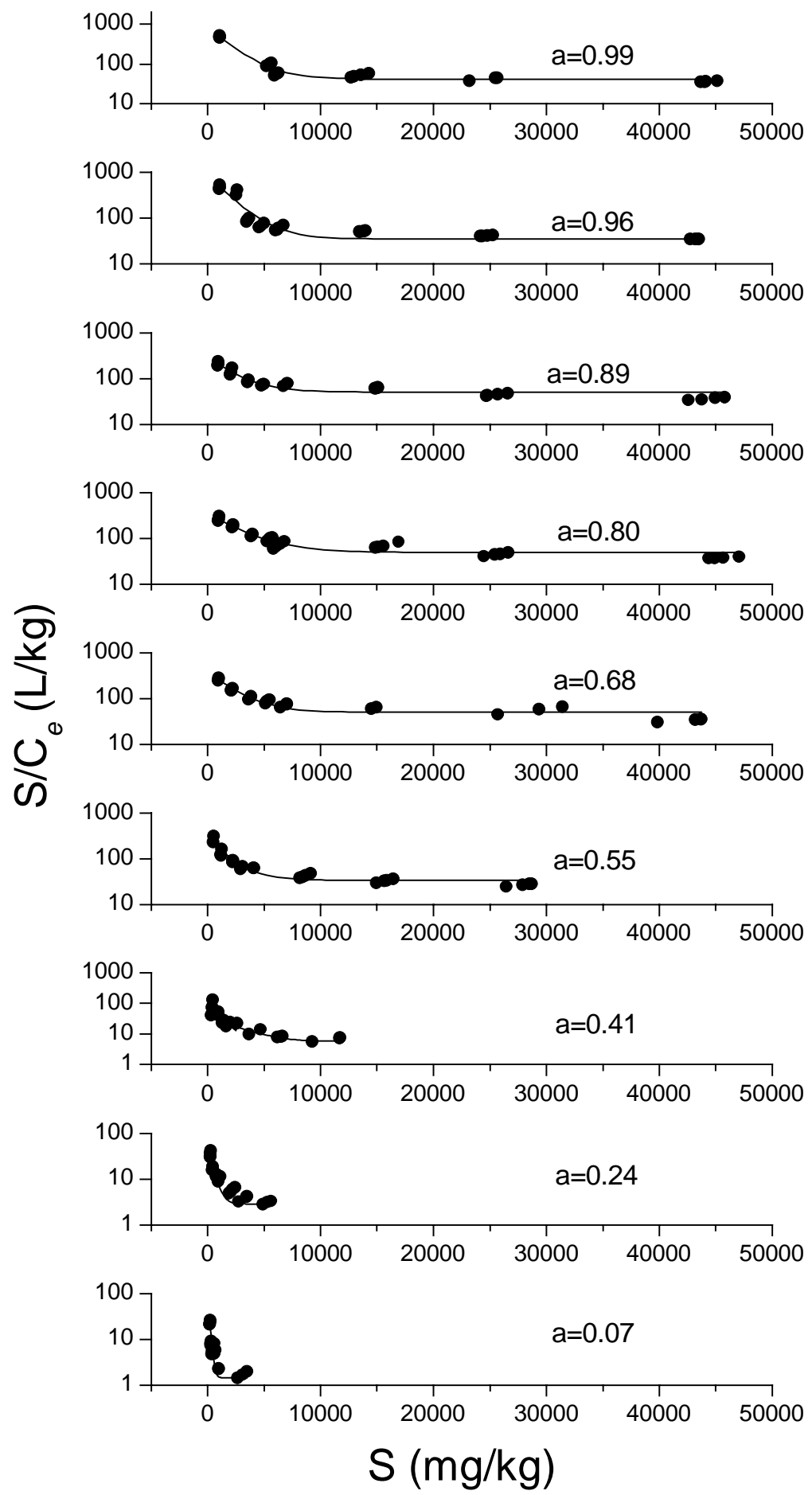

Fig. S3. $\mathrm{S} / \mathrm{C}_{e}$ vs. $\mathrm{S}$ for all nine experimental hydration levels. Water activities (a) are labeled. 